\title{
Percutaneous Electrocauterization (Electrosclerotherapy) to Treat Cutaneous Hemangioma- our experience in Apollo Hospitals Dhaka
}

\author{
MM Hosain ${ }^{1}$, MM Rahman², MM Momen ${ }^{3}$, AKM F Haque,
}

\begin{abstract}
Introduction

Percutaneous Electrosclerotherapy technique for the treatment of cutaneous hemangioma is introduced as an alternative and minimally invasive treatment mode. The aim of the study was to evaluate the response of percutaneous electrosclerotherapy on cutaneous hemangioma, as monotherapy or in adjunct to surgery.
\end{abstract}

\section{Materials and Methods}

Fourteen patients with 10 females and 04 males underwent percutaneous electrosclerotherapy for the treatment of cutaneous hemangioma mostly on head and neck in Plastic and Cosmetic Surgery department of Apollo Hospitals Dhaka from January 2009 to June 2010. Percutaneous Electrosclerotherapy was applied through a Colorado needle with an intention to cauterize the bulk of the lesion at the tip of the needle but not burning the skin. Pre and post operatively the size of the lesions were measured to see the percentage of regression.

\section{Results}

Percutaneous Electrosclerotherapy was done in all 14 cases. Most of the lesions (78\%) were smaller in size $(<5 \mathrm{~cm})$ and the site was mostly in head and neck (57\%), more than half of the patients (57\%) got significant benefit from this procedure. The regression of size is mostly in children $<10$ years which is noted in about $65 \%$.

The only complication found was overlying skin necrosis due to burn in 35\% of patients which was managed usually by simple dressing and antibiotics with complete healing.

\section{Conclusion}

Percutaneous Electrosclerotherapy is effective monotherapy for smaller hemangioma and prior to surgery to reduce the volume, operation time, bleeding, as well as post operative scar. It can be done easily with minimal hospital stay, less cost and complication.

\section{Key words}

Hemangioma, electrocauterization, electrosclerotherapy, monotherapy

\section{Introduction}

Hemangiomas vasoformative are immature nests of tissue that demonstrate angioblastic proliferation and regression and represent the most common vascular tumor of childhood. ${ }^{1}$ Infantile hemangioma is more common in females, which is about $10 \%$ of all children and usually appears within a few weeks after birth; about $30-90 \%$ of cases undergo a characteristic proliferation phase, followed by a stable phase and finally a period of regression or involution occurs ${ }^{2}$ which usually occurs between the ages of 10-12 years. ${ }^{3}$

Approximately $50 \%$ of hemangiomas involute 
by the age 5 years, $70 \%$ involute by the age 7 years, and $90 \%$ involute by the age 9 years. The later the involution more residual skin abnormality is likely.

Hemangiomas and vascular malformations are benign tumors with increased vascularity. ${ }^{4}$ The aim of the treatment is the removal of the vascular mass for both cosmetic and functional reasons. ${ }^{3}$ Although surgical treatment is the most prevalent method used, surgery alone is not sufficient for the treatment of large, extensive tumors and also in some special sites.

The search for alternative methods to reduce or eliminate vascularity of these lesions is directed towards occlusion of the vessels such as embolization or sclerotherapy. ${ }^{4}$ Actually several factors that come into play when deciding what treatment is best - whether the hemangioma is proliferating or involuting; whether it is superficial or deep/compound; the location of the lesion and the age of the patient.

In general common treatment options are- i) Observation or wait and see policy, ii) Medical treatment - considered only during the fast growth stage of the hemangioma, most commonly used drugs are steroids and less commonly used agent is interferon 2 alpha which can be given both orally or intralesionally but later one has limited benefit, the typical lesions that respond to injection are small, circumscribed and deep. iii) Laser- pulse dye laser (PDL) with dynamic cooling device for superficial vascular hemangiomas; NdYAG laser for deep hemangiomas; KTP laser for airway lesions, CO2 and Erbium laser for resurfacing to scar revisions and improving the texture of skin. iv) Surgery- In involution phase and may be in the late proliferative phase (Large lip hemangioma that has not responded to steroid) v) Less commonly used methods are -Electrothrombosis (Electrosclerotherapy), embolotherapy, cryotherapy, compression or tattooing etc. These treatments may be used singly or in combination to treat hemangioma.
The percutaneous electrocauterization technique for the treatment of cutaneous hemangioma is introduced as an alternative and minimally invasive treatment mode. In this method percutaneously a small sheathed needle was used where it is possible to do needle point monopolar electrocauterization at the tip only due to the insulation of the other part of the needle. Gently changing the direction or site of the needle we can approach whole bulk of the hemangioma. Caution should be taken not to burn the vital organ or overlying skin. As it is a simple procedure we can do it as a day case under local anesthesia or under general anesthesia. It can be done as a monotherapy for small hemangioma or prior to surgery to reduce the volume and size as well as fear for bleeding.

\section{Materials and Methods}

From January 2009 to July 2010, 14 patients with cutaneous hemangioma in different sites were treated with percutaneous Electrosclerotherapy in Apollo Hospitals Dhaka. Among them 10 patients were females and 04 were males. The age of the patients ranged from 3 years to 27 years. All patients needed more than one session. We used Colorado needle [fig- 1] that was fixed with a monopolar diathermy to cauterize the lesion. Most of them were done under General Anesthesia (10 cases) very few were under Local Anesthesia (04 cases). Preoperative and post operatively the size of the hemangioma was measured in which the diameter ranged from 3 $\mathrm{cm}$ to $15 \mathrm{~cm}$ before operation. Preoperatively patient was assessed for anesthesia and feasibility for operation.

\section{Technique}

After desired anesthesia, antiseptic wash and draping was done. A very small stab incision was made at one side of the lesion through which the needle tip was introduced to the main bulk of the abnormal vascular tissue keeping the insulating part at the skin margin to assure that 


\section{ORIGINAL ARTICLE}

no skin will burn during the procedure. The needle tip was then gently moved in all directions to achieve the maximum volume to be cauterized. After cauterization no dressing was given in smaller cases just applying some antibiotic ointment over the area, but in larger cases pressure bandage was given. In some cases overlying skin necrosis was seen which was treated with dressing, antibiotics and excision of the area with primary closure.

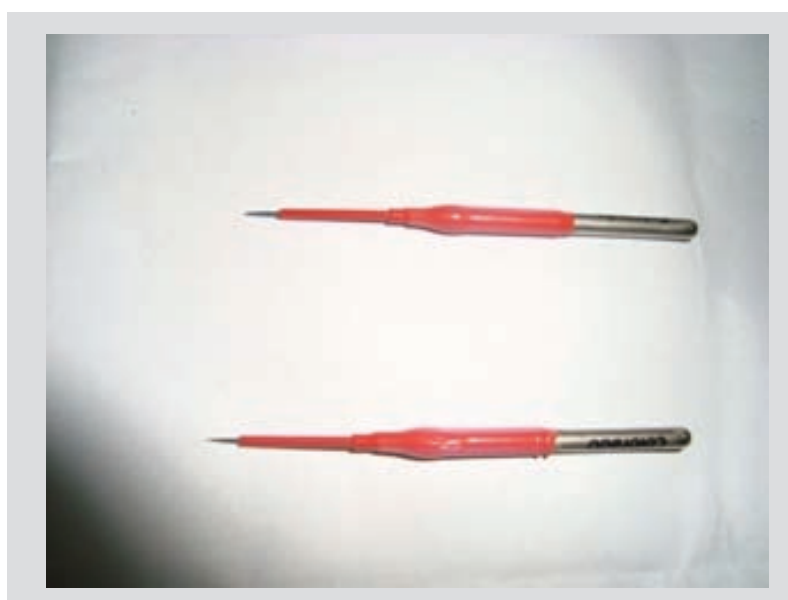

Fig 1: Colorado needle

\section{Results}

In this study 14 patients with different size of hemangioma were treated with percuteneous electrosclerotherapy, where most of them were $<5 \mathrm{~cm}$ (11 cases) only 03 of them were $>5 \mathrm{~cm}$. About $2 / 3$ of the patients (10 cases) were children $<10$ years [Fig-4, 5]

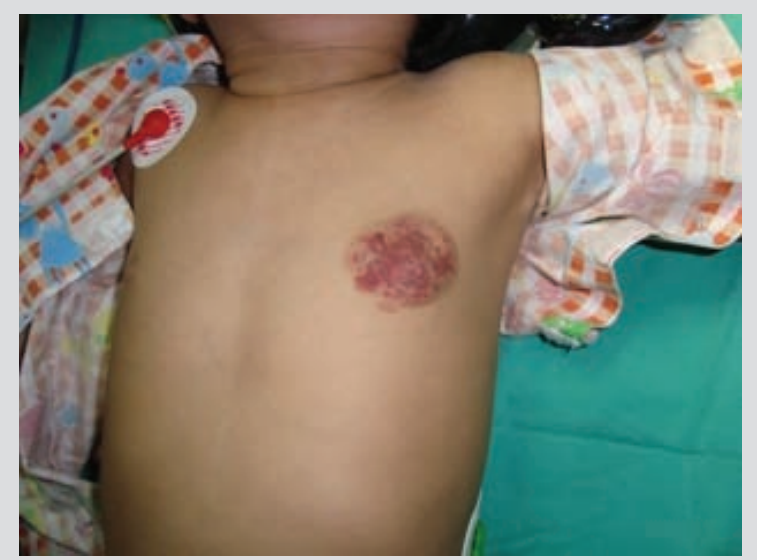

Fig 4: A patient with hemangioma of left breast before $3^{\text {rd }}$ session

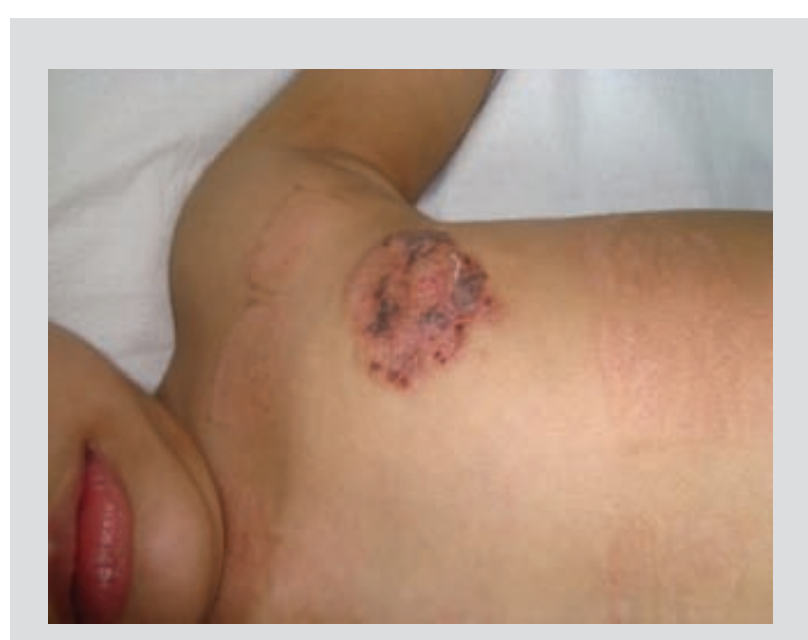

Fig 5: A Patient with hemangioma of left breast- after $3^{\text {rd }}$ session

and only 04 cases were $>10$ years. [Table-1]

Table 1: Age and sex distribution

\begin{tabular}{|l|l|l|l|l|l|}
\hline Age distribution & \multicolumn{2}{|l|}{} & \multicolumn{3}{|c|}{ Sex distribution } \\
\hline$<10$ years & 10 & $71 \%$ & Female & 10 & $71 \%$ \\
\hline$>10$ years & 04 & $29 \%$ & Male & 04 & $29 \%$ \\
\hline
\end{tabular}

The head and neck bears the most common site (08 cases) [Fig-2], 05 patients having it in the trunk and only 01 had hemangioma in the extremity. [Table-2]

Table 2: Site distribution of hemangioma

\begin{tabular}{|l|l|l|}
\hline Site & No of patients & Percentage \\
\hline Head and neck & 08 & $57 \%$ \\
\hline Trunk & 05 & $35.7 \%$ \\
\hline Extremity & 01 & $7.3 \%$ \\
\hline
\end{tabular}

Females were predominant than male counterpart. (Female- 10 cases, male-04 cases)[Table-1] 
Electrosclerotherapy alone was satisfactory for 09 cases [Fig-2, 3];

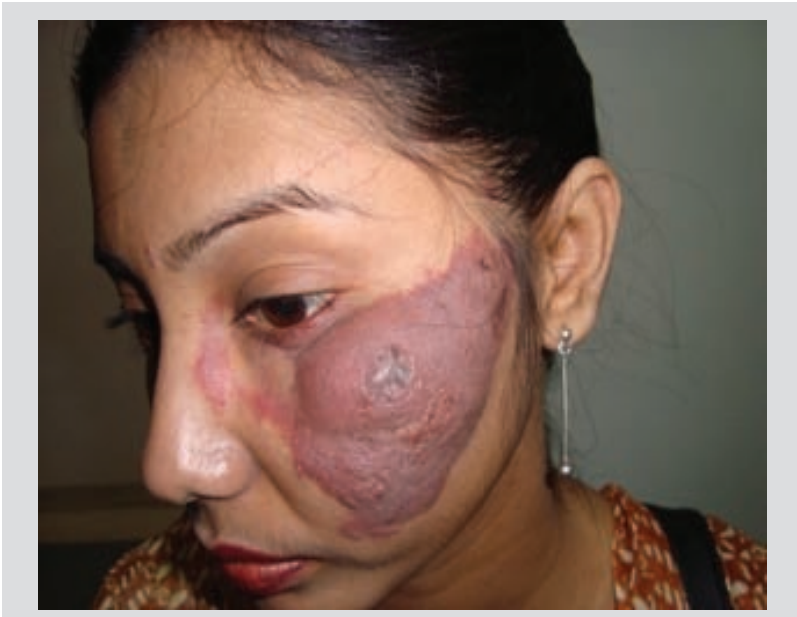

Fig 2:Patient with hemangioma face - prior to procedure

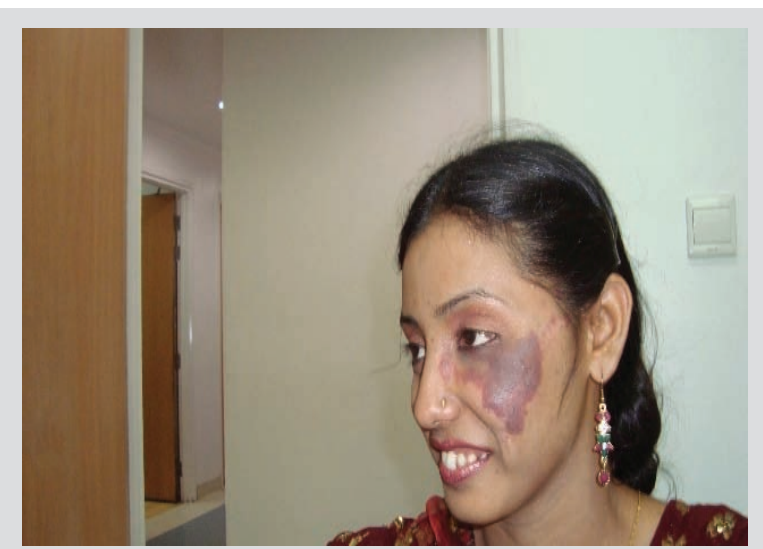

Fig 3: Patient with hemangioma face -after the procedure

05 cases needed surgery as a combination with it. The regression is satisfactory in < 10years group that is about $65 \%$ where as $45 \%$ in older group. [Table-3]

Table 3: Percentage of regression according to the age group and number of cases

\begin{tabular}{|l|l|l|}
\hline Age group & \% of regression & No of patient \\
\hline$<10$ Years & $65 \%$ & 10 \\
\hline$>10$ years & $45 \%$ & 04 \\
\hline
\end{tabular}

Nearly all patients needed 03 sessions only 02 cases needed 05 sessions.
In all cases volume reduction was satisfactory but some patients needed surgery to get satisfactory results.

The only complication is overlying skin necrosis due to burn of the skin but it was found in 05 cases which was treated conservatively or excised to reduce the patient's morbidity or bad scar.

\section{Discussion}

We have 14 patients with cutaneous hemangioma who were selected to be treated with Percutaneous Electrosclerotherapy with common age group for children $<10$ years which is about $71 \%$ of total cases. This result also agreed with the study of Winter et al, who found $65.3 \%$ of patients in child group. ${ }^{3}$

The male female ratio in our study was about 2:1 and that is similar to the study of Saleh $\mathrm{KH}^{5}$ and supported by Garzon $\mathrm{M}$ who found the ratio $3: 1{ }^{6}$

Most common sites we found was head and neck $(>57 \%)$ which is agreeable with Saleh KH (51\%) ${ }^{5}$ and Mulkin and Glowacki (60\%).

The size that we found in most cases ( $>78 \%$ ) was smaller $(<5 \mathrm{~cm})$ that was also found in Garzon's report $(83.3 \%){ }^{6}$

The treatment response is satisfactory for all cases as it reduces the cost, operation times, tumor size and volume as well as simplify the surgical procedures that agrees with Erol OO et $\mathrm{al}^{8}{ }^{8} 08$ of the patients that were benefited significantly, of them 04 cases indicated they generally improved and 2 stated they have benefited little. Remarkable percentage of regression (65\%) of tumor was seen in children $<10$ years age group which was better compared to the Saleh study with medical sclerotherapy who found $50 \%$ regression.

\section{Conclusion}

Percutaneous electrosclerotherapy is an effective procedure for cutaneous hemangioma as 


\section{ORIGINAL ARTICLE}

monotherapy or prior to surgery. It is cost effective as well as simple procedure, can do as a day case. Most of the patients with

cutaneous hemangioma can be benefited by this simple, minimally invasive procedure.

\section{References}

1. Akyuz C. management of cutaneous hemangioma. Pediatr Hematol Oncol. 2001;18:47-55. [PubMed]

2. Sadan N. Treatment of infants with high doses of prednisolone. J Pediatr. 1996;128:141-6. [PubMed]

3. Winter H, Drager E, Sterry W, Sclerotherapy in treatment of hemangioma. Dermatol surg. 2000;26:105-8. [PubMed]
4. C. Karaca, A. Baruteu, M. Yilmaz, M. Akguner, A. Menderes. Treatment of hemangioma and vascular malformation by electrothrombosis. Eur J Plast Surg. 1997; 20:192-196. [PubMed]

5. Saleh KH. The steroid benefit of treating complicated hemangioma. Indian $\mathrm{J}$ Plast Surg. 2009;42(2):242-4. [PubMed]

6. Garzon M. Hemangioma update on classification. Cutis. 2000;66:325-83. [PubMed]

7. Mulkin, Glowacki. Hemangioma and vascular malformation of the head and neck. 1989;34:156-98.

8. Erol OO, Uysal OA, Agaoglu Galip. Percutaneous Electrothrombosis: A minimally invasive technique for the treatment of deep hemangioma. Aesth Plast Surg.2010; 34(2) 214-7. 